\title{
Simple model for low-frequency guitar function
}

\section{Christensen, Ove; Vistisen, Bo B.}

\section{Published in:}

Acoustical Society of America. Journal

Link to article, DOI:

10.1121/1.384814

Publication date:

1980

\section{Document Version}

Publisher's PDF, also known as Version of record

Link back to DTU Orbit

Citation (APA):

Christensen, O., \& Vistisen, B. B. (1980). Simple model for low-frequency guitar function. Acoustical Society of America. Journal, 68(3), 758-766. https://doi.org/10.1121/1.384814

\section{General rights}

Copyright and moral rights for the publications made accessible in the public portal are retained by the authors and/or other copyright owners and it is a condition of accessing publications that users recognise and abide by the legal requirements associated with these rights.

- Users may download and print one copy of any publication from the public portal for the purpose of private study or research.

- You may not further distribute the material or use it for any profit-making activity or commercial gain

- You may freely distribute the URL identifying the publication in the public portal

If you believe that this document breaches copyright please contact us providing details, and we will remove access to the work immediately and investigate your claim. 


\title{
Simple model for low-frequency guitar function
}

\author{
Ove Christensen \\ Institute of Medical Physiology A, University of Copenhagen, 3 C, Belgdamsvej, 2200 Copenhagen N. Denmark
}

\begin{abstract}
Bo B. Vistisen
The Acoustics Laboratory, Technical University of Denmark, 2800 Lyngby, Denmark

(Received 8 November 1979; accepted for publication 25 May 1980)

The frequency response of sound pressure and top plate mobility is studied around the two first resonances of the guitar. These resonances are shown to result from a coupling between the fundamental top plate mode and the Helmholtz resonance of the cavity. A simple model is proposed for low-frequency guitar function. The model predicts frequency responce of sound pressure and top plate mobility which are in close quantitative agreement with experimental responses. The absolute sound pressure level and mobility level are predicted to within a few decibels, and the equivalent piston area of the top plate is determined. All parameters of this model can directly be derived from measurements of the frequencies of the two first resonances and of the cavity Helmholtz resonance. The Helmholtz resonance is found as the antiresonance in the spectrum of top plate mobility.
\end{abstract}

PACS numbers: 43.75.De

\section{INTRODUCTION}

The two lowest resonance frequencies of the guitar occur at approximately 100 and $200 \mathrm{~Hz}$. These resonances are found in all guitars and may vary in frequency in the typical ranges $90-120 \mathrm{~Hz}$ for the first resonance and $170-250 \mathrm{~Hz}$ for the second resonance. Hologram interferometric studies of the guitar top plate $^{1}$ have shown that the second resonance corresponds in frequency to the lowest (fundamental) mode of the top plate. The first resonance is found only in complete instruments, i.e., when a cavity enclosure is added to the top plate. It is therefore related to the Helmholtz resonance of the guitar cavity.

The open strings on the guitar are tuned at $E_{2}(82.4 \mathrm{~Hz})$ for the lowest and at $E_{4}(329.6 \mathrm{~Hz})$ for the highest pitched string. The response of the instrument in the frequency range of the two first resonances is therefore important for the musical quality of the instrument. $^{2}$ For the viol in family, Hutchins ${ }^{3}$ has shown the importance of the two lowest resonances, "air" and "main wood," in determining the musical properties of the complete instrument, and she has used the scaling properties of these resonances with instrument size to construct a new family of violins. We have therefore found it worthwhile to study the guitar in the frequency range of the two lowest resonances.

In this work we consider the frequency response of the sound pressure and of the top plate mobility (mechanical admittance) of the guitar in the range of the two first resonance frequencies. The coupling between the Helmholtz resonance and the top plate fundamental resonance is investigated and the relation between these resonances and the first two resonances of the guitar is given.

A simple model is presented for this system of coupled oscillators on the basis of Newtonian equations of motion. All parameters of the model are determined experimentally. In spite of its simplicity, the model correctly describes the variation with frequency of the sound pressure level and the top-plate mobility level. Furthermore, the absolute value of these quantities are predicted to within a few decibels.

\section{METHODS AND RESULTS}

\section{A. Experimental procedure}

All measurements were performed in an anechoic chamber. The guitar was usually mounted horizontally on a pad of rockwool to prevent vibrations of the back. In a few cases, the guitar was mounted with the back free to oscillate on three soft plastic foam blocks, one under the neck and two placed symmetrically under the edges of the guitar at the lower bout. The exciter used was made of a small loudspeaker with the membrane removed. The mass of the exciter should be small to avoid mass loading of the top plate if true resonance frequencies are wanted. Also, the losses of the exciter should be small to avoid errors in measurement in the vicinity of resonances.

The lowest vibration mode of the guitar is symmetrical and essentially all top plate movement takes place in the lower bout of the guitar. ${ }^{1}$ The second top plate mode occurs usually at $240-300 \mathrm{~Hz}$ and consists of an antisymmetrical vibration of the two halves of the top plate in the lower bout. The guitar was excited at the center of the bridge to avoid significant contribution from the second top plate mode. A B \& K heterodyne analyzer, type 2010 , supplied the signal to the exciter through a power amplifier and measured the signal from either microphone or accelerometer. Frequency was swept in the range $60-300 \mathrm{~Hz}$ and output continously recorded on a $B \& K$ type 2307 recorder. The microphone, $B \& K$ type 4117 , was placed $2 \mathrm{~m}$ above a point on the top plate between the soundhole and bridge. This distance is sufficiently large to insure that the far field from the instrument is measured in the frequency range studied. The accelerometer, B \&K type 4344 , 
was mounted as close as possible to the exciting point at the center of the bridge. In order to measure force, a $B \& K$ force transducer, type 8200 , was used. The force was kept constant at about $0.2 \mathrm{~N}$ by means of a compression loop to the $\mathbf{2 0 1 0}$ analyzer. The force transducer adds a mass load of $21 \mathrm{~g}$ to the top plate which changes the resonances of the guitar. The force transducer was therefore only used in studies concerning the general physical behavior of the guitar.

Usually, it is fairly easy to measure resonance frequencies of mobility or radiation. However, when measurements are to be compared with theoretical predictions, much care is needed to eliminate minor artifactual resonances stemming from mounting of guitar or transducer. This is particularly important because the mobility and sound pressure levels vary some 40 $\mathrm{dB}$ in the frequency range studied.

\section{B. Experimental study of the two first resonances}

The purpose of the experiments reported in this section is to study the relation between the first two resonances of the guitar, denoted $f_{-}$and $f_{+}$, and the Helmholtz (cavity) and the fundamental top plate resonances. This can be done by selectively changing either the top plate resonance frequency or the Helmholtz resonance frequency and observing the effect of this on the two first resonances. The top plate resonance frequency was changed by mass loading the top plate with weights $(20-50 \mathrm{~g})$ placed next to the point of excitation. The Helmholtz resonance frequency was changed by placing a "collar" into the soundhole. The collar was made of a strip of cardboard which was formed into the shape of a cylinder $3-4 \mathrm{~cm}$ high, fitting the soundhole. In this way, the effective length of the air volume in the soundhole is increased without modifying soundhole diameter or cavity volume. This leads to a lowering of the Helmholtz resonance frequency.
In Fig. 1 are shown spectra of top plate mobility and of corresponding sound pressure level for a guitar. The upper set of curves represent the normal situation. The sound pressure shows the two first resonances at $f_{\text {- }}$ $=103 \mathrm{~Hz}$ and at $f_{+}=198 \mathrm{~Hz}$. The top plate mobility dis plays resonances at the same frequencies but shows also an antiresonance between the first and second resonance at $126.5 \mathrm{~Hz}$. When the top plate frequency is lowered by mass loading with $39.3 \mathrm{~g}$, the first two resonances move to $f_{-}=100.5 \mathrm{~Hz}$ and $f_{4}=173 \mathrm{~Hz}$, but the antiresonance stays at the same frequency. Although the shift of resonance frequencies in this experiment is most pronounced for the second resonance, the shift of $2.5 \mathrm{~Hz}$ to lower frequencies of the first resonance is significant. A similar shift was observed on four other guitars. The fact that both resonances shift when the top plate frequency is lowered shows that the first and second resonances are coupled. When the Helmholtz frequency is lowered (third set of curves of Fig. 1) the first resonance moves to $f_{-}=87 \mathrm{~Hz}$, the second to $f_{+}=196 \mathrm{~Hz}$, and the antiresonance is lowered to 110.5 $\mathrm{Hz}$. The effect of changing the Helmholtz resonance is thus most markedly on the first resonance although there is a significant frequency shift of the second resonance too.

The antiresonance is thus changed when the Helmholtz resonance is changed and remains unchanged when the top plate resonance is changed. It is thus clear that this antiresonance represents the Helmholtz resonance. We shall accordingly denote the antiresonance frequency by $f_{h}$.

The first two resonances of the guitar are thus shown to be a result of the interaction of the Helmholtz resonance and the fundamental top plate resonance. For a pure Helmholtz resonator, the air piston in the orifice oscillates against the stiffness of the air in the cavity. In the guitar, the top plate vibration results in small
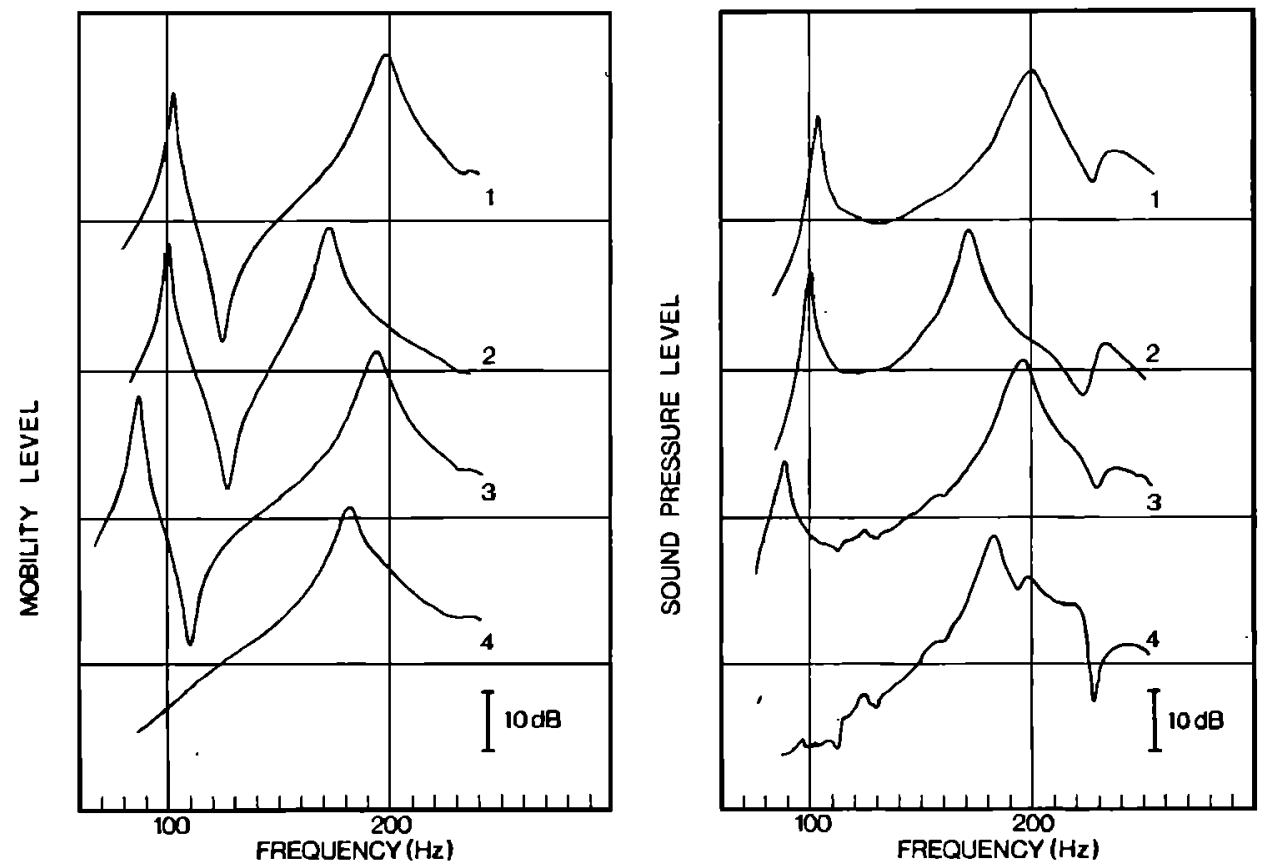

FIG. 1. The effect of specific changes in top plate resonance frequency and in Helmholtz frequency on mobility level of top plate (left panel) and sound pressure level (right panel). Mobility level is defined as $20 \mathrm{log}$ (mobility/reference mobility). The four sets of curves represent, in order from top to bottom (1) normal guitar; (2) top plate loaded with $39.3 \mathrm{g;}$ (3) "collar" inserted in soundhole; (4) soundhole plugged with wooden disk. Curves are displaced 25 dB for clarity. The horizontal lines through (the lower part of) each curve represent in the left panel a mobility level of $-45 \mathrm{~dB}$ re $1 \mathrm{mN}^{-1} \mathrm{~s}^{-1}$, and in the right panel a sound pressure level of $55 \mathrm{~dB}$, measured 2 $\mathrm{m}$ from the guitar In response to an exciting force of $0.2 \mathrm{~N}$. 
periodic variations in cavity volume which leads to periodic compression of the air in the cavity. The pressure variations in the cavity will in turn excite the air piston in the soundhole so that a coupling is established between the top plate resonance and the Helmholtz resonance. If the soundhole is plugged, as shown in the lower set of curves in Fig. 1, the Helmholtz resonance disappears and the pure uncoupled top plate resonance, $f_{p}$, is seen. This resonance occurs in the present guitar at $184 \mathrm{~Hz}$. It should be noted that this frequency represents the resonance of the top plate with the stiffness of the air in the cavity added. The resonance frequency of the top plate without cavity, $f_{p, 0}$, is less than $f_{p}$. It is, however, noteworthy that the interaction in the guitar takes place between the Helmholtz resonance and the resonance of the top plate backed by the cavity.

Thus the net result is, that the uncoupled resonances, at frequencies $f_{h}=126.5 \mathrm{~Hz}$ and $f_{p}=184 \mathrm{~Hz}$, interact to result in the first and second resonance at 103 and 198 $\mathrm{Hz}$. It is a general result from the theory of coupled oscillators, to be developed for the guitar in the following section, that the square sum of the resonance frequencies in the coupled system equals the square sum of resonance frequencies in the uncoupled system, i.e.,

$$
f_{+}^{2}+f_{-}^{2}=f_{b}^{2}+f_{h}^{2} \text {. }
$$

Taking the values of $f_{-}, f_{+}$, and $f_{k}$ from the upper mobility spectrum of Fig. 1 , we calculate $f_{p}=183 \mathrm{~Hz}$, in ag reement with the observed value on the plugged guitar.

The Helmholtz frequency for the present guitar is $126.5 \mathrm{~Hz}$. For other guitars we found values in the range $122-135 \mathrm{~Hz}$. The frequency predicted on the basis of a volume of 13 liters and a soundhole diameter of $88 \mathrm{~mm}$ is $142 \mathrm{~Hz}$. It has been shown, that in actual guitar-shaped cavities, the Helmholtz frequency depends also on the position of the soundhole in the gui$\operatorname{ta} x^{4}$ and on the height of the sides. ${ }^{5}$ In a normal guitar, both effects tend to lower the Helmholtz resonance relative to the one predicted from volume and soundhole diameter.

The occurrence of a coupling between Helmholtz and the first top plate resonance was first realized by Meyer, ${ }^{4}$ who measured the resonance frequency of the dismounted top plate and of the rigid wall cavity and compared these with the two first resonances in the assembled instrument. The approach presented here has the advantage that the Helmholtz frequency can be determined on the complete instrument from a measurement of the top plate velocity.

\section{SIMPLIFIED MODEL FOR LOW-FREQUENCY GUITAR FUNCTION}

\section{A. Basic assumptions}

The simplified model for guitar function in the frequency region around the two first resonances is shown in Fig. 2. It is obvious from the figure, that many simplifying assumptions have been made. The fundamental top plate mode in a real guitar is characterized by a symmetrical variation of amplitude over the lower

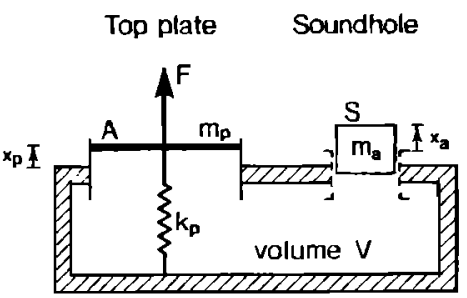

FIG. 2. Simplified model for guitar function at low frequencies. The top plate is replaced by a piston of equivalent area $A$ and mass $m_{p}$, connected to a spring of stiffness $k_{p}$. The Helmholtz resonator is modeled as an air piston of mass $m_{a}$, oscillating against the stiffness of the air in the cavity.

bout of the guitar. This continuous system is in the present model replaced by a simple harmonic oscillator consisting of a plate of mass $m_{p}$ and piston area $A$, together with a spring of stiffness $k_{\mathrm{p}}$. The resonance frequency of the top plate without any cavity is therefore $\omega_{b, 0}=\left(k_{b} / m_{b}\right)^{1 / 2}$. Clearly, both the mass and the area of the oscillator must be thought of as equivalent values which will have to be defined from experiments. The top plate is acted upon by a harmonically varying force $F$ which in the experiments is supplied by the exciter. The Helmholtz resonator consists of an air piston of mass $m_{\mathrm{a}}$ in the soundhole oscillating against the stiffness of the air in the cavity. The bottom of the guitar is in this analysis assumed to be rigid. Basically, this system acts as a reflex enclosure. ${ }^{6.7}$ The symbols used in the following are explained in Table $I$.

\section{B. Derivation of the physical model}

The position of the top plate at a given time is $x_{p}$, relative to its equilibrium position and the position of the air piston is $x_{a}$. Both of these are assigned positive values for outward movement. The corresponding change in the volume of the cavity on Fig. 2 is $\Delta V=A x_{p}+S x_{a}$. For adiabatic compression, the resulting change in cavity pressure is $\Delta p=-\mu \Delta V$, where we define

$$
\mu=c^{2} \rho / V \text {. }
$$

This pressure excerts a force on the two oscillators of $A \Delta p$ and $S \Delta p$. Thus a displacement of one oscillator induces a change in cavity pressure which gives rise to a force on the other oscillator. This basically explains the coupling between the first top plate resonance and the Helmholtz resonance. In order to calculate the pressure $\Delta p$ correctly, the volume displacement $x_{p} A$ should equal the actual volume displacement produced by the top plate. If we let $x_{p}$ represent the maximum displacement at the center of the bridge, $A$ will accordingly be smaller than the actual area of the lower bout of the guitar. We can now write the equations of motion for the two oscillators as:

$$
\begin{aligned}
& m_{p} \ddot{x}_{p}=F-k_{p} x_{p}-R_{p} \dot{x}_{p}+A \Delta p, \\
& m_{d} \ddot{x}_{a}=S \Delta p-R_{a} \dot{x}_{a},
\end{aligned}
$$

where $R_{b}$ and $R_{a}$ are the resistances to motion of the plate and air piston. For the real guitar the equivalent area in the term $A \Delta p$ might not exactly equal the area giving the correct volume displacement. For the sake of simplicity, we shall only use one symbol for the 
TABLE I. Symbol explanation. The general symbol for frequency is $f$ and corresponding angular frequency is $\omega$. The upper part of the table are experimentally observable quantities, the lower part are model parameters which are derived from these. Quantities marked with $*$ can be derived from unmarked quantities. Top plate resonance refers to the fundamental, or first vibrational mode of the top plate.

\begin{tabular}{|c|c|c|}
\hline Symbol & Unit & Description \\
\hline \multicolumn{3}{|r|}{ Observable quantities } \\
\hline$c$ & $\mathrm{~ms}^{-1}$ & sound velocity in air, $343.3 \mathrm{~ms}^{-1}$ \\
\hline$\rho$ & $\mathrm{kg} \mathrm{m}^{-3}$ & density of air, $1.205 \mathrm{~kg} \mathrm{~m}^{-3}$ \\
\hline$F$ & $\mathrm{~N}$ & force of excitation \\
\hline$f$ & $\mathbf{s}^{-1}$ & first resonance frequency of guitar \\
\hline$f_{+}$ & $\cdots$ & second resonance frequency of guitar \\
\hline$f_{h}$ & $\cdots$ & $\begin{array}{l}\text { Helmholtz resonance frequency of } \\
\text { guitar cavity }\end{array}$ \\
\hline$x_{p}, x_{a}^{*}$ & $\mathbf{m}$ & displacement of top plate and air piston \\
\hline$u_{p}, u_{a}^{*}$ & $\mathrm{~ms} \mathrm{~s}^{-1}$ & velocity of top plate and air piston \\
\hline$p$ & $\mathrm{~N} \mathrm{~m}^{-2}$ & $\begin{array}{l}\text { sound pressure at distance } R \text { from guitar; } \\
\text { sound pressure level, re } 20 \mu \mathrm{Pa} \text { is given in decibels. }\end{array}$ \\
\hline $\boldsymbol{R}$ & $\mathrm{m}$ & distance from guitar top plate to microphone \\
\hline$V$ & $\mathrm{~m}^{3}$ & volume of guitar cavity \\
\hline$\mu^{*}$ & $\mathrm{~N} \mathrm{~m}^{-5}$ & $\begin{array}{l}\text { proportionality constant between changes } \\
\text { in volume and pressure, } \Delta p=\mu \Delta V\end{array}$ \\
\hline$\Delta p *$ & $\mathrm{~N} \mathrm{~m}^{-2}$ & $\begin{array}{l}\text { adiabatic change in cavity pressure due to } \\
\text { movement of top plate and air piston. }\end{array}$ \\
\hline$m_{p}$ & $\mathrm{~kg}$ & equivalent top plate mass \\
\hline$Q_{-}, Q_{+}$ & & quality factors at $f_{-}$and $f_{+}$ \\
\hline$\gamma * \gamma_{+}^{*}$ & $\operatorname{rad~s}^{-1}$ & 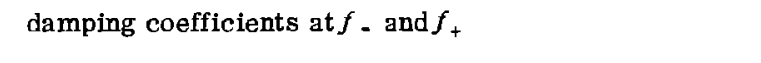 \\
\hline & & Model parameters \\
\hline$f_{p}$ & $s^{-1}$ & $\begin{array}{l}\text { top plate resonance frequency in } \\
\text { uncoupled system }\end{array}$ \\
\hline$f_{p, 0}$ & $\cdots$ & resonance frequency of free top plate \\
\hline$f_{p h}$ & & coupling frequency \\
\hline$f_{a}$ & $\cdots$ & $\begin{array}{l}\text { resonance frequency of top plate as acoustically } \\
\text { suspended membrane in guitar cavity } \\
\text { with soundhole closed, i.e., value of } \\
f_{p} \text { if } k_{p}=0 \text {. }\end{array}$ \\
\hline$k_{p}^{*}$ & $\mathrm{~N} \mathrm{~m}^{-1}$ & stiffness of top plate \\
\hline$A$ & $\mathbf{m}^{2}$ & equivalent piston a rea of top plate. \\
\hline$s$ & $\cdots$ & area of soundhole \\
\hline$m_{a}^{*}$ & $\mathbf{k g}$ & mass of air piston in soundhole \\
\hline$\alpha^{*}$ & $\mathrm{~N} \mathrm{~m}^{-1}$ & $\begin{array}{l}\text { stiffness constant for coupling between } \\
\text { top plate and Helmholtz oscillators, } \alpha=\mu A S\end{array}$ \\
\hline$R_{p}^{*}, R_{a}^{*}$ & $\mathrm{~N} \mathrm{sm}^{-1}$ & $\begin{array}{l}\text { resistance to movement of top plate and } \\
\text { air piston in uncoupled system. }\end{array}$ \\
\hline$\gamma_{b}^{*}, \gamma_{a}^{*}$ & $\operatorname{rad~s}{ }^{-1}$ & $\begin{array}{l}\text { damping coefficients of top plate and air } \\
\text { piston in uncoupled system, } \gamma_{p}=R_{p} / m_{p} \text { and } \\
\gamma_{a}=R_{a} / m_{a}\end{array}$ \\
\hline
\end{tabular}

equivalent area. When in the above equations we insert $\Delta p=-\mu\left(x_{p} A+x_{a} S\right)$ the following result is obtained:

$$
\begin{aligned}
& m_{p} \ddot{x}_{p}=F-x_{p}\left(k_{p}+\mu A^{2}\right)-R_{p} \dot{x}_{p}-\mu S A x_{a} \\
& m_{a} \ddot{x}_{a}=-\mu S^{2} x_{a}-R_{a} \dot{x}_{a}-\mu S A x_{p} .
\end{aligned}
$$

The last term in these equations gives the coupling between the two oscillators. The coupling can be expressed in terms of a coupling constant of the same dimension as a spring constant

$$
\alpha=\mu S A \text {. }
$$


It is seen from Eq. (1a) that the plate resonance in the closed cavity $(S=0)$ is

$$
\omega_{p}=\left[\left(k_{p}+\mu A^{2}\right) / m_{p}\right]^{1 / 2} \text {. }
$$

For zero spring constant, the plate frequency would be

$$
\omega_{a}=\left(\mu A^{2} / m_{b}\right)^{1 / 2}
$$

which is the resonance frequency of an acoustically suspended membrane closing a cavity of volume $V$. The relation between these frequencies is

$$
f_{p}^{2}=f_{b, 0}^{2}+f_{a}^{2},
$$

where $f_{p .0}$ is the top plate resonance measured without cavity.

With the plate clamped $\left(x_{p}=0\right)$, Eq. (1b) gives the Helmholtz resonance frequency

$$
\omega_{h}=\left(\mu S^{2} / m_{a}\right)^{1 / 2}
$$

If the effective length of the air oscillating in the soundhole is $l_{\text {eff }}$, one obtains the usual relation

$$
\omega_{h}=c\left(S / V l_{\mathrm{eff}}\right)^{1 / 2} \text {. }
$$

To solve Eqs. (1) for a harmonically varying force, we let $F \rightarrow F \exp (i \omega t)$ and similarly for $x_{p}$ and $x_{a}$. From Eq. (1b) we then express $x_{a}$ in terms of $x_{p}$ and substitute this relation into Eq. (1a). Since we are more interested in the velocities $u_{p}$ and $u_{a}$ of the plate and the air piston we state the results for these, using $\gamma_{p}=R_{p} / m_{p}$ and $\gamma_{a}=R_{a} / m_{a}$ :

$$
\begin{aligned}
& u_{p}=i \omega\left(F / m_{p}\right)\left\{\left[\left(\omega_{h}^{2}-\omega^{2}\right)+i \omega \gamma_{p}\right] / D\right\}, \\
& u_{a}=-i \omega\left(F / m_{p}\right)(A / S)\left(\omega_{h}^{2} / D\right),
\end{aligned}
$$

where the denominator $(D)$ is given by

$$
D=\left(\omega_{p}^{2}-\omega^{2}+i \omega \gamma_{p}\right)\left(\omega_{h}^{2}-\omega^{2}+i \omega \gamma_{h}\right)-\omega_{p h}^{4} .
$$

Here, the coupling frequency $\omega_{p h}$ is given by

$$
\omega_{p h}^{4}=\alpha^{2} / m_{p} m_{\alpha}=\omega_{h}^{2} \omega_{\alpha}^{2} .
$$

In the following we make the usual assumption that losses are so small that they do not influence the resonance frequencies. The resonances of the system occur when the denominator

$$
\left(\omega_{p}^{2}-\omega^{2}\right)\left(\omega_{h}^{2}-\omega^{2}\right)-\omega_{p h}^{4}
$$

is zero. This happens at the frequencies $\omega_{-}$and $\omega_{+}$, the first and second resonances of the guitar, which are given by

$$
\omega_{ \pm}^{2}=\frac{1}{2}\left(\omega_{b}^{2}+\omega_{h}^{2}\right) \pm \frac{1}{2}\left[\left(\omega_{p}^{2}-\omega_{h}^{2}\right)^{2}+4 \omega_{p h}^{2}\right]^{1 / 2} .
$$

As a corollary to this relation, we observe that

$$
\omega_{+}^{2}+\omega_{-}^{2}=\omega_{b}^{2}+\omega_{h}^{2}
$$

as anticipated in the preceeding section. As a result of the coupling, $f_{-}$is smaller than $f_{h}$ and $f_{+}$is larger than $f_{p}$. The physical reason for this is that at $f_{-}$, the air piston moves in phase with the backside of the top plate and at $f_{+}$, in antiphase.

The denominator can be written as

$$
D=\left(\omega_{+}^{2}-\omega^{2}+i \omega \gamma_{+}\right)\left(\omega_{-}^{2}-\omega^{2}+i \omega \gamma_{-}\right) .
$$

The damping coefficients $\gamma_{-}$and $\gamma_{+}$, which apply to the first and second resonances can, after some lengthy but trivial calculations, be shown to be given by

$$
\begin{aligned}
& \gamma_{+}=[(1+G) / 2 G]\left\{\gamma_{p}+[(G-1) /(G+1)\}_{a}\right\}, \\
& \gamma_{-}=[(1+G) / 2 G]\left\{\gamma_{a}+[(G-1) /(G+1)] \gamma_{p}\right\}, \\
& G=\left(\omega_{+}^{2}-\omega_{-}^{2}\right) /\left(\omega_{p}^{2}-\omega_{a}^{2}\right) .
\end{aligned}
$$

Thus the coupling between the two oscillators is reflected as a coupling between the losses at the two first resonances.

In order to calculate the farfield of the radiated sound pressure from the guitar, we note that in the frequency range of interest here, the wavelength of the sound is considerably larger than the typical dimensions of the lower bout of the guitar. It is therefore reasonable to approximate the acoustical radiation with that from a simple source. The sound pressure at distance $R$ from a simple source radiating in $4 \pi$ solid angle is

$$
p=-i \omega \rho U / 4 \pi R \text {, }
$$

where $U$ is the total volume velocity of the source. In the present case, both top plate and the air in the soundhole contribute to the volume displacement so that $U=A u_{p}+S u_{a^{*}}$. When the velocities from Eqs. (6) are inserted in this equation, one obtains for the sound pressure:

$$
p=-(i / 4 \pi)(\rho / R)\left(A / m_{p}\right) F\left(\omega^{4} / D\right) .
$$

Both plate velocity (mobility) and the sound pressure show the two resonances at $f_{-}$and $f_{+}$. However, for the top plate mobility, there is an antiresonance between the first two resonances. This agrees with the experimental findings of Fig. 1. At frequencies less than $f_{h}$, the-two oscillators move $180^{\circ}$ out of phase [Eqs. (6)] in such a manner that the volume displaced by the top plate is short circuited through the soundhole. This gives rise to a rapid decline of sound pressure towards low frequencies in agreement with experiment. The top plate and air piston have opposite phases below the Helmholtz resonance. Above this resonance, , they have the same phase and in this way the guitar acts as a reflex enclosure. Over the whole frequency range, the fractional contributions of the two oscillators to the sound pressure vary as $\left(f_{h}^{2} / f^{2}-1\right)$ for the top plate and as $-f_{h}^{2} / f^{2}$ for the soundhole [Eqs. (6)]. The soundhole gives the major contribution at low frequencies and the top plate dominates in the high frequency range. The presence of the Helmholtz resonance below the first top plate resonance thus significantly extends the range of the instrument towards lower frequencies.

The top plate exhibits a characteristic phase shift of $180^{\circ}$ at $f_{-}$and another shift of $180^{\circ}$ back to the previous value at $f_{h}$ as shown in Fig. 5 .

\section{Evaluation of model parameters from experiment}

In this section we show how the parameters introduced in the preceeding section can be evaluated from experimental data. The most important input data are the frequencies of the two first resonances and the Helmholtz resonance, all of which can be obtained from measurement of top plate mobility. 
We start by assuming known values of $f_{-}, f_{h}$, and $f_{+}$. Then $f_{p}$ can be found from Eq. (9) and the coupling frequency $f_{p h}$ can be found from Eq. (8). Relation (7) is then used to find the frequency $f_{a}$ from which Eq. (4) yields the free top plate frequency $f_{p, 0}$ -

The equivalent top plate mass $m_{p}$ is determined by observing the change in $f_{p}$ when the top plate is loaded with a known mass (cf. Fig. 1) and subsequent use of Eq. (2). In the present experiments, the mass load is placed close to the exciter and velocity transducer at the center of the bridge. This procedure is common practice and has been used on instruments of the viol in family. ${ }^{9}$ When $m_{p}$ is known, the top plate stiffness can be calculated from the value of $f_{b .0^{*}}$ Usually, mass calculations are based on the variation of $f_{+}$with load (rather than $f_{p}$ ). Such calculations include the coupling between the two oscillators. This leads to an overestimate of $m_{p}$ for guitars of typically $30 \%$.

The equivalent piston area $A$ can now be determined from the calculated value of $f_{a}$ [Eq. (3)] together with the measured value of the cavity volume. As mentioned in the last section, there are for an actual instrument two, in principle different, equivalent areas. The first is the area that gives the correct volume displacement, $A x_{p}$. The second is the one that gives the correct force $A \Delta p$. The equivalent piston area determined from Eq. (3) is the mean proportional between these.

In this way, all parameters determining the response of the guitar are known with the exception of the damping coefficients $\gamma_{+}$and $\gamma_{-} \cdot \gamma_{-}$can be found from measurement of the frequency difference $\Delta f$ - between the $3-\mathrm{dB}$ limits of the first resonance in the sound pressure level using $\gamma_{-}=2 \pi \Delta f_{-}$. Similarly for $\gamma_{+}$, mutatis mutandis. Furthermore, $\gamma_{a}$ is obtained from $\gamma_{+}$and $\gamma_{-}$ by inverting the relations (10). $\gamma_{a}$ is used in the nominator of the plate mobility.

Instead of using damping coefficients, we use in the next section the quality factors, i.e., $Q_{-}=f_{-} / \Delta f_{-}$in accordance with common practice.

\section{COMPARISON BETWEEN EXPERIMENT AND THEORY}

\section{A. Information from measurements of $f_{-}, f_{h}$, and $f_{+}$}

In this section, we shall assess some typical values of the parameters determining the instrument response.

The most important result of the last chapter is that the first top plate resonance and the Helmholtz resonance form a pair of coupled harmonic oscillators. As a result of this coupling, the resonance frequencies are shifted to what become the first and second resonances of the instrument. The relation between the two sets of frequencies is given in Eq. (8). In order to represent this relation graphically it is convenient to normalize all frequencies in $\mathrm{Eq} .(8)$ by $f_{\mathrm{h}}$. Using dashed variables for normalized frequencies, this relation becomes

$$
f_{ \pm}^{\prime 2}=\frac{1}{2}\left(f_{p}^{\prime 2}+1\right) \pm\left[\left(f_{p}^{\prime 2}-1\right)^{2}+4 f_{p h}^{\prime 4}\right]^{1 / 2} \text {. }
$$

In Fig. 3 we have shown $f_{+}^{\prime}$ and $f_{-}^{\prime}$ as functions of nor- malized plate frequency $f_{p}^{\prime}$ with coupling frequency ratio $f_{p h}^{\prime}$ as parameter. For zero coupling frequency ratio, one obtains $f_{+}^{\prime}=f_{b}^{\prime}$ and $f_{-}^{\prime}=1$ which is represented by the two straight lines in the figure. When $f_{D h}$ differs from zero, the resultant frequencies are $f_{+}$, which is always larger than $f_{h}$ and $f_{-}$, which is always smaller than $f_{h}$. As a result of the coupling, the degeneracy at $f_{b}=f_{h}$ is removed so that for all instruments one should observe $f_{-}, f_{h}$, and $f_{+}$in the mobility spectrum. A plot similar to Fig. 3 was used by Ägren ${ }^{9}$ and by Meyer. ${ }^{4}$ The latter author show however $f_{-}=0$ and $f_{+}=f_{h}$ at $f_{p}=0$ which is in contradiction to the present findings. This has, however, little consequence for normal instruments for which $f_{p}>f_{h}$.

In Fig. 3 are also shown experimentally obtained values for $f_{+}^{\prime}, f_{-}^{\prime}$ versus $f_{p}^{\prime}$. The small dots in the figure are data from Meyer. ${ }^{4}$ These data represent 10 different guitar top plates which were gradually stiffened by adding transverse bars and fan bracing. This gives a total of 54 "different" top plates which were measured in a cavity with $f_{h}=139 \mathrm{~Hz}$. Twenty-nine of these plates were also measured in a cavity with $f_{h}$ $=125.6 \mathrm{~Hz}$, yielding a total of 83 sets of data points.

It is seen from the figure that the coupling frequency ratio $f_{p h}^{\prime}$ varies from about 0.7 to 0.9 for the instruments studied. Apparently, the coupling increases with increasing top plate frequency. Notice that the coupling between the two oscillators is strong so that the resultant resonance frequencies deviate significantly from the original ones.

In order to make the reader familiar with typical orders of magnitude, we take a characteristic example. Assume that $f_{-}=100 \mathrm{~Hz}, f_{h}=123 \mathrm{~Hz}$, and $f_{+}=214 \mathrm{~Hz}$. Using the procedure described in the preceeding section

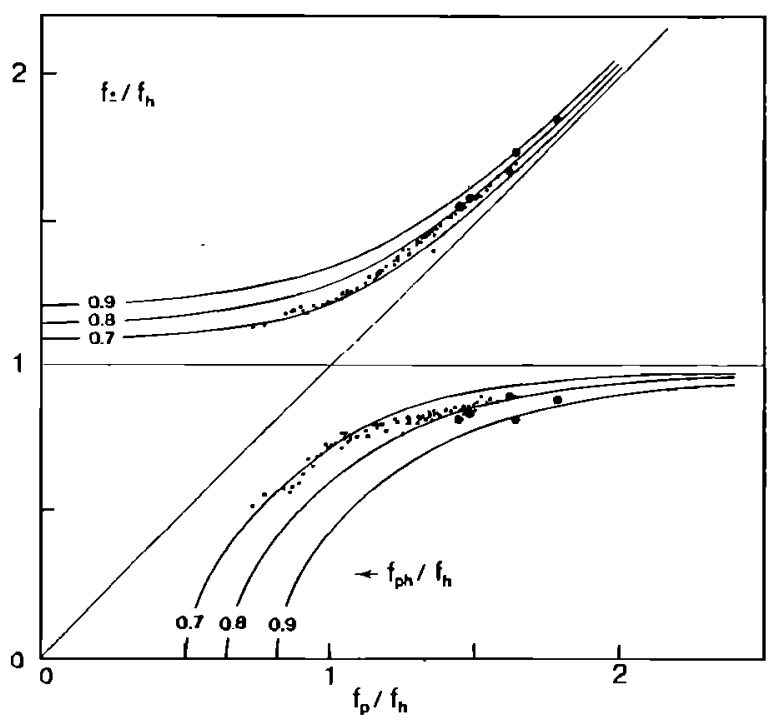

FIG. 3. The two first resonance frequencies $f_{*}$ (upper set of curves) and $f_{-}$(lower set of curves) plotted as function of top plate resonance frequency, $f_{p}$. All frequencles are normallzed with respect to the Helmholtz frequency, $f_{h}$. The curves are

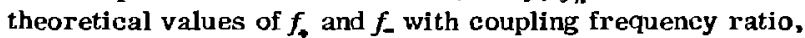
$f_{b h} / f_{h}$, as parameter. The large points are experimental frequencies from the present work, the small points are experimental data by Meyer.4 
we calculate $f_{p}=201.7 \mathrm{~Hz}, f_{p h}=112 \mathrm{~Hz}, f_{p, 0}=174 \mathrm{~Hz}$, and $f_{a}=102 \mathrm{~Hz}$. Values of top plate equivalent mass are from 60 to $110 \mathrm{~g}$ as determined on four guitars. Taking a value of $80 \mathrm{~g}$ and a cavity volume of 13 liters, we calculate $k_{b}=1.3 \times 10^{5} \mathrm{~N} / \mathrm{m}$ and an equivalent piston area of $A=550 \mathrm{~cm}^{2}$. On two class ic guitars we measured a total top plate area of $1400 \mathrm{~cm}^{2}$ for both. The areas in the lower bout of the instrument, delimited by the transverse bar, were 865 and $900 \mathrm{~cm}^{2}$, respectively. Although caution should be taken aga inst a too literal interpretation of equivalent mass and piston area, it is rather satisfying to be able to calculate a reasonable piston area on the basis of data of so simple a nature.

The sound pressure from an instrument is directly proportional to $A / m_{\rho}$. The coupling frequency $f_{p h}$, as well as the frequency $f_{a}$, increase with the size of the ratio $A^{2} / m_{b}$. One can from these relations draw the conclusion that a good instrument is characterized by a large coupling. Since the Helmholtz frequency usually does not vary much from instrument to instrument, this leads to the rough conclusion, that good instruments have rather low values of $f_{-}$. For the most highly appreciated instruments we have studied, this frequency was found in the range $92-102 \mathrm{~Hz}$. It should of course not be forgotten that good instruments are characterized not only by their performance at low frequencies.

\section{B. Frequency variation of mobility level and sound pressure level}

In Fig. 4 is shown experimental data for variation of top plate mobility and sound pressure level versus frequency. Also shown are theoretical points based on Eqs. (6a) and (11). The only data used in this calculation are $f_{-}, f_{h}, f_{+}, Q_{+}, Q_{-}$, and $m_{p}$ together with the cavity volume $V$. Both for mobility and for sound pressure level, the theoretical predictions show a frequency variation in neat accordance with the measured spectra. The same parameters were used to calculate mobility and sound pressure level (SPL). Notice that the absolute value for mobility and SPL is calculated in the figure.

A correspondance between experimental findings and theoretical expectations as it is presented in Fig. 4 was obtained for all guitars investigated. If we include experiments with variation of top plate and Helmholtz frequencies, a total of about ten experiments have been compared with theoretical calculations. The fit in Fig. 4 is representative for the agreement between theory and experiments.

Below the first resonance and above the second resonance, deviations between theory and experiment occur. At low frequencies, this can be attributed to contributions from resonances in the mechanical support for guitar or exciter. Above the second resonance, deviations between theory and experiment can be attributed to the second top plate resonance, which on Fig. 4 is seen around $280 \mathrm{~Hz}$. Due to this resonance, the measured mobility is smaller than that calculated
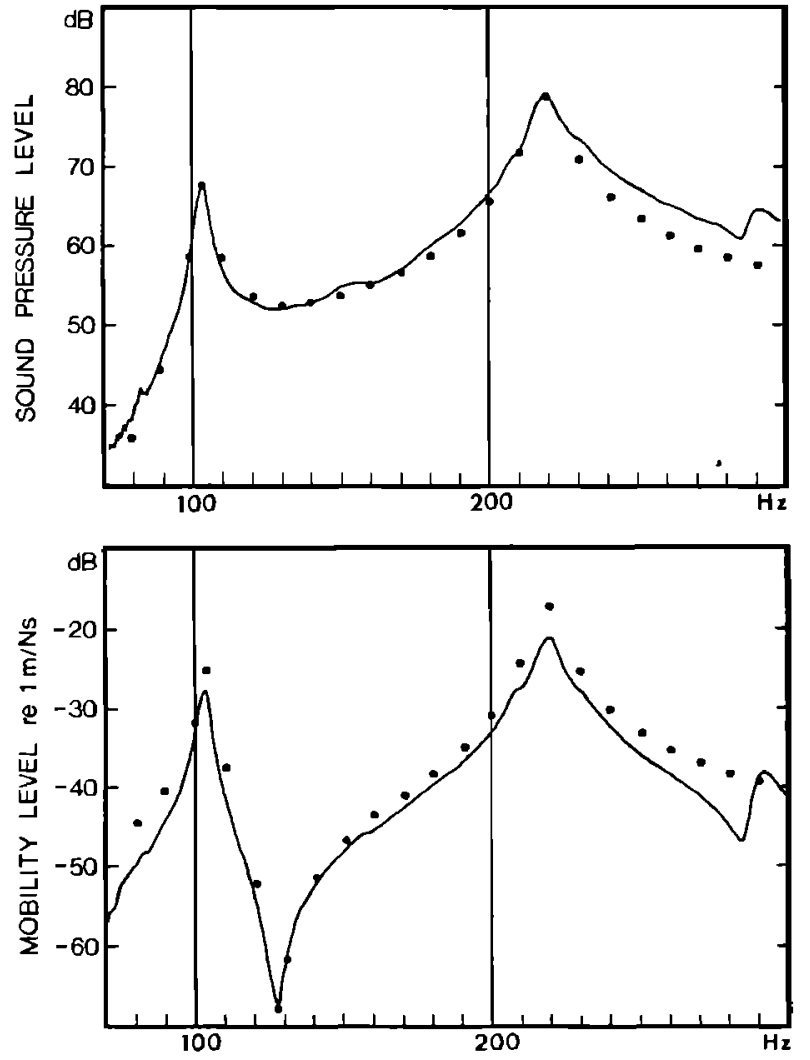

FIG. 4. Comparison between experimental results and theoretical predictions of sound pressure level and top-plate mobility level. The points are calculated frequency responses. The data used in the calculation of both responses are: $f_{-}=104$ $\mathrm{Hz}, f_{h}=127 \mathrm{~Hz}, f_{+}=219 \mathrm{~Hz}, m_{p}=112 \mathrm{~g}, Q_{-}=29.0$, and $Q_{+}=25.8$. The sound pressure level was calculated at $2 \mathrm{~m}$ distance from the guitar top plate in response to a force of $0.2 \mathrm{~N}$. Notice that absolute levels are calculated.

since the first and second top plate resonances oscillate out of phase at frequencies between the resonances. Usually, a contribution to the sound pressure from the second top plate mode is rather small since this resonance acts as a dipole.

The best frequency region to compare theory and experiment is between $f_{h}$ and $f_{+}$. Both for SPL and mobility, there is a $80-100 \mathrm{~Hz}$ region where the spectra in practice are independent of the losses. This allows us to compare absolute values of experimental data with theory. The result of such a comparison is that the absolute level of sound pressure is correctly determined but the mobility level is overestimated by $2-4 \mathrm{~dB}$. Notice that the experimental mobility represents the masimum mobility measured at antinode of the top plate.

The significance of this is that the top plate equivalent mass seems to be underestimated by $2-4 \mathrm{~dB}$ but that the ratio of equivalent area to mass seems to be correctly estimated by the procedure described in Sec. IIC.

In Fig. 5 is shown the phase of the top plate mobility. The guitar is the same as shown in Fig. 4 but the measurement was performed three months before the one in Fig. 4. The calculated phase is from Eq. (6a). The phase exhibits a drastic $180^{\circ}$ change between $f_{-}$and $f_{k}$ 


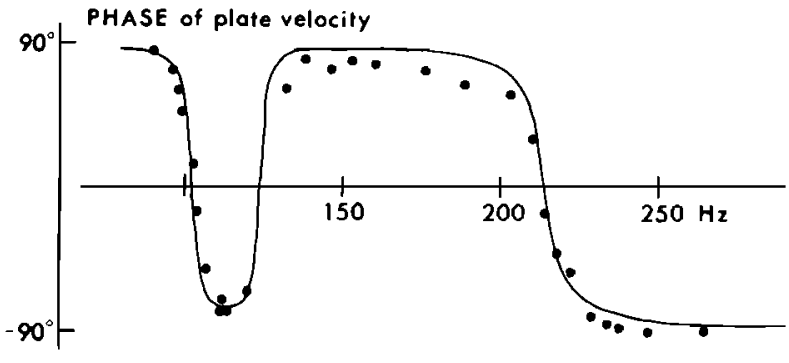

FIG. 5. Frequency variation of phase of top plate velocity relatlve to exciting force. Points are experimental data and the curve is calculated from theory. The data used in the calculation are: $f_{-}=102 \mathrm{~Hz}, f_{h}=124 \mathrm{~Hz}, f_{+}=214 \mathrm{~Hz}, Q_{-}=29.0$, and $Q_{+}$ $=25.8$.

as predicted by theory. Measurements were not possible around $f_{h}$ due to the very small signal level here. The resonance frequencies in $\mathrm{Fig}$. 5 differ $2-4 \mathrm{~Hz}$ from the ones in Fig. 4. Such variations were commonly observed and presumably reflect changes in the instrument with environmental conditions.

The change in phase between $f_{-}$and $f_{h}$ is perhaps the most direct experimental evidence for the existence of a coupling between the top plate and Helmholtz resonances.

Determination of the quality factor $Q$ can be performed by measurement of 3-dB limits of the resonance in the sound pressure spectrum. We have, however, used a method based on measurement of the peak sound pressure level relative to the minimum between the first two resonances. Algebraic manipulations with Eq. (11) show that the frequency at the minimum, $f_{\min }$, is given by

$$
f_{\text {min }}^{2}=2 f_{+}^{2} f_{-}^{2} /\left(f_{+}^{2}+f_{-}^{2}\right),
$$

and that the sound pressure here is

$$
p_{\min }=F \frac{i}{4 \pi} \frac{\rho}{R} \frac{A}{m_{p}} \frac{4 f_{+}^{2} f_{-}^{2}}{\left(f_{+}^{2}-f_{-}^{2}\right)^{2}},
$$

which is independent of losses. The sound pressure at the resonances $f_{-}$and $f_{+}$depends on $\gamma_{-}$and $\gamma_{+}$and on the factor $F A /\left(R m_{p}\right)[\mathrm{Eq} .(11)]$. This factor is substituted from the experimental value of $p_{\min }$ given in Eq. (14) so that $\gamma_{-}$and $\gamma_{+}$can be found and hence $Q_{-}$and $Q_{+}$.

Quality factors were evaluated after this method on nine guitars, four of which were made by luthiers of international reputation. On two guitars, $Q_{+}$could not be determined due to the closeness of the second top plate resonance to $f_{+}$. The values found were $Q_{+}=24.3$ $\pm 5.6(15.5$ to 34.8$)$ and $Q_{-}=16.5 \pm 6.7(6.5$ to 29$)$. The better guitars had highest values of $Q_{\text {. }}$.

The damping coefficients at $f_{+}$and $f_{-}$are linear combinations of the damping coefficients at $f_{p}$ and $f_{h}$, according to Eqs. (10). These relations can be used to find $Q_{p}$ and $Q_{a}$. The general result is, based on five guitars, that $Q_{p}$ is slightly less than $Q_{+}$and that $Q_{a}$ is us ually larger than $Q_{\text {_. }}$ The highest value for $Q_{-}$is 48.

It is interesting that even in the extreme case of negligible losses in the Helmholtz resonator the losses at $f_{-}$are nonzero. For the numerical example in Sec. II one would get $Q_{-}=56$ if $Q_{+}$is chosen as 20 .

The losses are partly radiation losses and partly internal losses. The radiation resistance $R_{\mathrm{rad}}$ for a piston of area $A$, is

$$
R_{\mathrm{rad}}=\pi f^{2} \rho A^{2} / c
$$

which is valid for a simple source radiating in $4 \pi$ solid angle. Using the relation (5) to obtain $S^{2} / m_{a}$ from $f_{h}$ and the relation (3) to obtain $A^{2} / m_{p}$ from $f_{a}$, the $Q$ values due to radiation losses at $f_{h}$ and $f_{p}$ can be found to be

$$
\begin{aligned}
& Q_{a}=c^{3} / 2 \pi^{2} V f_{h}^{3} \\
& Q_{p}=c^{3} / 2 \pi^{2} V f_{p} f_{a}^{2} .
\end{aligned}
$$

If we insert typical values in these equations $\left(f_{h}=123\right.$ $\mathrm{Hz}, f_{b}=201.7 \mathrm{~Hz}, f_{a}=102 \mathrm{~Hz}$, and $V=13$ liter) we get the quality factors $Q$ due to pure radiation losses to be $Q_{a}$ $=85.2$ and $Q_{b}=75.5$. It appears thus, that about one third to one fourth of the observed losses can be attributed to radiation losses.

The minimum sound pressure level between the resonances can be used to evaluate experimental figures for the ratio of equivalent piston area to equivalent mass [cf. Eq. (14)]. This ratio is directly proportional to the output from the instrument as low frequencies. The mean for nine guitars is $A / m_{p}=8.6 \pm 3.7 \mathrm{~cm}^{2} / \mathrm{g}$. The highest figure is 14.8 and the lowest is 1.9 . It is our opinion, that the first guitar is far better than the second in the low range, i.e., up to about $300 \mathrm{~Hz}$. A high ratio can in principle be obtained by using a thin top plate, but this in turn lowers the frequencies of the higher top plate modes which determine the treble response. The three highest rated instruments have $A / m_{p}$ ratios of 8.4 to 11.2 which probably can be taken as a practical compromise.

\section{DISCUSSION}

It has been shown in Sec. III, that the simple model developed in Sec. II satisfactorily predicts the low-frequency variation of top plate mobility and the SPL. We thus take it for proven that the first two resonances of the guitar are the result of the interacting top plate and Helmholtz resonances. The same conclusion was qualitatively reached by Meyer ${ }^{4}$ and by Ågren.9 Some of the components of the model developed here is also found in the work of Firth ${ }^{6}$ and of Caldersmith. ${ }^{7}$ In this work we have established a quantitative description of guitar function at low frequencies in which all parameters, with the exception of losses, can be derived directly from measurements of the frequencies of the two first resonances and of the Helmholtz resonance. In the literature, it is very common that the first resonance, the "air resonance," is called the Helmholtz resonance. This is not correct, the Helmholtz resonance lies in between the two first resonances in the SPL spectrum and can be easily determined from measurements of the top plate velocity.

We have in the derivation of the model assumed a rigid bottom plate. If this is not the case, one should instead consider a system of three coupled oscillators. Meyer 4 
has given a detailed technical description of this system. The effect of an oscillating bottom plate is to lower $f_{-}$and $f_{+}$and to split $f_{+}$in two resonances. This effect diminishes with increasing bottom plate frequency. until finally the splitting of $f_{+}$cannot be seen. The effect of the bottom can be seen in Fig. 1 in the SPL spectrum as an additional resonance around $230-40 \mathrm{~Hz}$. This guitar was mounted so that the bottom was free to oscillate. No effect of the bottom is seen in Fig. 4, where oscillations of the bottom plate were heavily damped.

The fact that the very simple model estimates the absolute mobility level to within 2-4 dB and the absolute sound pressure level even better, is an additional merit of the model which allows estimation of equivalent piston area. The values of equivalent piston area are $50 \%-60 \%$ of the top plate area in the lower bout of the guitar. This figure compares well with the results of Caldersmith $^{7}$ who measured the displacement of the top plate and found a mean displacement equal to $52 \%$ of the maximum displacement at the center of the bridge.

The coupling between the first top plate mode and the Helmholtz resonance should be found in other string instruments as, for instance, the violin family although one cannot without measurement know the importance of this coupling. The second resonance in the violin family, the "main wood" of "prime wood" resonance, lacks the symmetry found in the guitar due to the presence of the bass bar and the sound post. However, the only condition for establishment of a coupling is that the Helmholtz resonance is present and that the vibration of the top plate (plus back plate for instruments with a sound post) generates a net volume displacement. The coupling is therefore also present in members of the violin family.

In order to get a feeling for how the formalism developed in this paper works for the violin, we use the mobility data given for a violin by Firth. ${ }^{10}$ For this instrument, $f_{-}=278$ and $f_{+}=457 \mathrm{~Hz}$. The minimum of top plate velocity at $f_{h}$ is not so deep as for the guitar but can be found at approximately $295 \mathrm{~Hz}$. We can then calculate the following parameters: $f_{b}=446 \mathrm{~Hz}, f_{\rho h}$ $=186 \mathrm{~Hz}$, and $f_{a}=117.3 \mathrm{~Hz}$. Thus the coupling frequency measured relative to $f_{h}$ is 0.63 which is smaller that the typical value of 0.8 for the guitar. This is probably due to the presence of the sound post which reduces ${ }^{10}$ the volume displacement level of the violin by about 10 dB. Taking a volume of 1.5 liters we can calculate the ratio $A^{2} / \mathrm{m}$ to be $570 \mathrm{~cm}^{4} / \mathrm{g}$. This figure can be compared with the data of Schelleng ${ }^{\mathbf{a}}$ for a different violin: $m_{p}=17.8 \mathrm{~g}$ and $A=170 \mathrm{~cm}^{2}$ yielding $A^{2} / m_{p}=1620 \mathrm{~cm}^{4} / \mathrm{g}$. Considering that the data are obtained on different instruments, this is a fair agreement.
It is apparent from Eqs. (11) and (6c), that the frequency dependence of the SPL is completely determined by the first two resonances at $f_{-}$and $f_{+}$. One can read these frequencies directly off the SPL spectra without performing mobility measurements. However, if $f_{h}$ is determined from mobility measurements we have shown that considerable insight can be gained into the anatomy of the resonances and that valuable data, such as estimates of equivalent piston area and top plate mass can be obtained.

Finally, it should not be forgotten that actual instruments are excited by strings and not by constant-force transducers. The force on the bridge from a plucked string has a large number of harmonic components of level comparable to the fundamental. Under-normal playing conditions the force level is almost constant up to the third to sixth harmonic component, depending on where the string is plucked. Therefore it should not be forgotten that the sound from a guitar in the low-frequency range is greatly influenced by resonances of the top plate at higher frequencies.

\section{ACKNOWLEDGMENTS}

The authors wish to express their gratitude to Dr. J. Meyer, who kindly put his original data at our disposal and to recitalist Lars Trier, who kindly lent us four of his best instruments.

${ }^{1}$ E. V. Jansson, "A Study of Acoustical and Hologram Interferometric Measurements of the Top Plate Vibrations of a Guitar," Acustica 25, 95-99 (1971).

${ }^{2} J$. Meyer, "Die Bestimmung von Qualitätskriterien bei Gitarren," Das Musikinstrument No. 9 (1976).

${ }^{3} \mathrm{C}$. M. Hutchins, “The Physics of Violins," Sci. Am. 207 (5), 78-93 (November 1962).

${ }^{4} \mathrm{~J}$. Meyer, "Die Abstimmung der Grundresonanzen von Gitarren," Das Musikinstrument No. 2 (1974).

${ }^{5}$ F. T. Dickens, “Inertance of the Guitar Soundhole," Catgut Acoust. Soc. Newsletter No. 29 (1978).

${ }^{6}$ I. M. Firth, "Physics of the Guitar at the Helmholtz and First Top-plate Resonances," J. Acoust. Soc. Am. 61, 588-593 (1977).

${ }^{7}$ G. Caldersmith, "Guitar as a Reflex Enclosure," J. Acoust. Soc. Am. 63, 1566-1575 (1978).

${ }^{8} \mathrm{~J}$. C. Schelleng, "The Violin as a Circuit," J. Acoust. Soc. Am. 35, 326-338 (1963).

${ }^{9} \mathrm{C}$. H. Ågren, "Experimental Findings Concerning CavityAssisted Resonances in Stringed Instruments With a Soundpost," Phys. Scripta 14, 179-186 (1976).

${ }^{10}$ T. Firth. "Mechanical Admittance Measurements on the Sound Post of the Violin, and Its Action," Acustica 36, 332-339 (1976/77). 